\title{
Can Doppler Flow Parameters of Carotid Stenosis Predict the Occurrence of New Ischemic Brain Lesions Detected by Diffusion-Weighted MR Imaging after Filter-Protected Internal Carotid Artery Stenting?
}

\author{
Y. Gunduz, R. Akdemir, L.T. Ayhan, and N. Keser
}

\begin{abstract}
BACKGROUND AND PURPOSE: Carotid angioplasty and stent placement are increasingly being used for the treatment of symptomatic and asymptomatic carotid artery disease. Carotid angioplasty and stent placement carry an inherent risk of distal cerebral embolization, precipitating new brain ischemic lesions and neurologic symptoms. Our purpose was to evaluate the frequency of new ischemic lesions found on diffusion-weighted imaging after protected carotid angioplasty and stent placement and to determine the association of new lesions with ICA Doppler flow parameters.
\end{abstract}

MATERIALS AND METHODS: Fifty-two patients (mean age, $68 \pm 11$ years) with 50\%-69\% ( $n=20$, group 1 ) and $\geq 70 \%$ ( $n=32$, group 2$)$ internal carotid artery stenosis underwent carotid angioplasty and stent placement with distal filter protection. DWI was performed before and 48 hours after carotid angioplasty and stent placement.

RESULTS: Thirty-three (63.4\%) patients showed new lesions. The average number of new postprocedural lesions was 3.4 per patient. Most of the postprocedural lesions were $<5 \mathrm{~mm}$ (range, 3-23 mm), cortical and corticosubcortical, and clinically silent. Group 2 had a significantly higher number of new lesions compared with group 1 ( $P<.001)$. A significant relationship was found between ICA Doppler flow parameters and the appearance of new lesions.

CONCLUSIONS: The appearance of new ischemic lesions was significantly related to the Doppler flow parameters, particularly peak systolic velocity.

ABBREVIATIONS: CAS = carotid artery stenting; $\mathrm{CCA}=$ common carotid artery; EDV = end-diastolic velocity; PSV = peak systolic velocity

$S^{t+a t a n}$ troke is the most common life-threatening neurologic disorder and the most important single cause of disability. ${ }^{1,2} \mathrm{Ca}-$ rotid artery stenosis, a major risk factor for stroke, and distal embolization, arising from degenerative breakdown or thrombotic occlusion of complex plaques, are important mechanisms of stroke in patients with atherosclerotic internal carotid artery stenosis. ${ }^{3-6}$ Duplex sonography is currently the principal and, undoubtedly, the most accurate noninvasive and inexpensive diagnostic technique available for the evaluation of internal carotid artery stenosis. It provides information about the presence and severity of carotid stenosis, the velocity and characteristics of blood flow, and plaque morphology. ${ }^{7-10}$

Carotid angioplasty and stent placement for severe internal carotid artery stenosis have been introduced as a safe alternative to

Received May 30, 2013; accepted after revision August 12.

From the Departments of Radiology (Y.G., L.T.A.) and Cardiology (R.A., N.K.), Sakarya University Medical Faculty, Sakarya, Turkey.

Please address correspondence to Yasemin Gunduz, MD, Department of Radiology, Sakarya University Medical Faculty, 54100, Sakarya, Turkey; e-mail: dryasemingunduz@yahoo.com

http://dx.doi.org/10.3174/ajnr.A3904 medical and/or surgical treatment in patients at high risk for surgical procedures. ${ }^{11,12}$ However, there is still a major concern regarding its safety because of the risk of distal cerebral embolization during the procedure. Recent technical refinements, therefore, have led to the widespread use of carotid artery stenting (CAS) with cerebralprotection devices, markedly reducing thromboembolic complication rates. ${ }^{13}$ Diffusion-weighted MR imaging is a very sensitive and specific technique for diagnosing cerebral ischemia. ${ }^{14,15}$ It has been used to detect structural damage of the brain due to cerebral embolism after cerebral angiography, neurointerventional procedures, and carotid endarterectomy. ${ }^{16,17}$

The purpose of our study was to assess, with DWI, the number, size, and location of new brain lesions after protected CAS and to evaluate the association of these new lesion deficits and Doppler flow parameters of ICA.

\section{MATERIALS AND METHODS Study Population}

From July 2010 to April 2013, 72 patients with sonographically significant lesions (diameter stenosis of $\geq 50 \%$ ) underwent elective carotid angiography and stent placement in the ICA (40 right, 
32 left), of which 52 consecutive patients were enrolled and 20 patients were excluded from this study.

The following conditions were exclusion criteria: patients who underwent an urgent carotid artery stent placement for severe neurologic symptoms; those with disabling stroke; lack of compliance; prior carotid intervention; contralateral ICA occlusion; history of heart failure and myocardial infarction 72 hours before CAS; significant thyroid, renal, or hepatic dysfunction; incomplete or poorquality MR imaging data; and any contraindication for MR imaging examination (pacemaker, claustrophobia).

The carotid angiograms were reviewed by an experienced cardiologist, who determined the severity of each lesion according to the criteria of the North American Symptomatic Carotid Endarterectomy Trial. ${ }^{18}$ According to our research protocol, the carotid artery was stented in asymptomatic patients with $\geq 80 \%$ stenosis of the extracranial carotid artery and in symptomatic patients with $\geq 70 \%$ stenosis.

Neurologic examination was performed before and after CAS by an experienced neurologist who was not involved in the CAS procedure. Patients were considered symptomatic in case of a transient ischemic attack, a retinal ischemic event, or an ischemic stroke within the territory of the narrowed carotid artery.

In addition, baseline risk factors for atherosclerosis, age, sex, hypertension, hyperlipidemia, diabetes mellitus, history of ischemic heart disease, and smoking were investigated for each patient.

The study, which complied with the Declaration of Helsinki, was approved by the local ethics committee, and all patients enrolled in the study gave written informed consent.

\section{Carotid Ultrasonography}

Baseline carotid ultrasonography was performed between 1 and 30 days before carotid angiography. All participants were examined after a 10-minute rest period to minimize the changes in blood pressure and heart rate to avoid influencing the Doppler parameters. Atherosclerosis of the carotid arteries was assessed by a radiologist who was blinded to the clinical data by using the same Doppler ultrasound device (Aplio MX; Toshiba Medical Systems, Tokyo, Japan). All patients were evaluated initially preprocedurally and postprocedurally with duplex ultrasonography of the ipsi- and contralateral common carotid artery (CCA) and ICA.

ICA atherosclerosis was evaluated by the maximum percentage of diameter reduction recorded by B-mode sonography and by the peak systolic velocity (PSV), end-diastolic velocity (EDV), and ICA/CCA PSV ratio per Doppler ultrasound. Lesion severity was defined as the greatest stenosis observed on either the right or left ICA. Sonography and Doppler findings were classified into 2 categories $^{19}$ :

1) Clinically insignificant: ICA stenosis of $<50 \%$ (normal and noncritical). PSV $<125 \mathrm{~cm} / \mathrm{s}$ with no signs of the presence of a sonographic atherosclerotic lesion (no plaque or intimal thickening is visible sonographically), correlating to additional criteria, including ICA/CCA PSV ratio $<2.0$ and ICA EDV $<40 \mathrm{~cm} / \mathrm{s}$.

2) Clinically significant: a) ICA stenosis of 50\%-69\% (PSV $=125-$ $230 \mathrm{~cm} / \mathrm{s}$ and the presence of a sonographic atherosclerotic lesion). Additional criteria include an ICA/CCA PSV ratio of 2.04.0 and ICA EDV of $40-100 \mathrm{~cm} / \mathrm{s}$. b) ICA stenosis of $\geq 70 \%$, but less than near-occlusion ( $\mathrm{PSV}$ is $>230 \mathrm{~cm} / \mathrm{s}$ and the presence of a sonographic atherosclerotic lesion). Additional criteria include an ICA/CCA PSV ratio $>4$ and ICA EDV $>100 \mathrm{~cm} / \mathrm{s}$ (Fig 1).

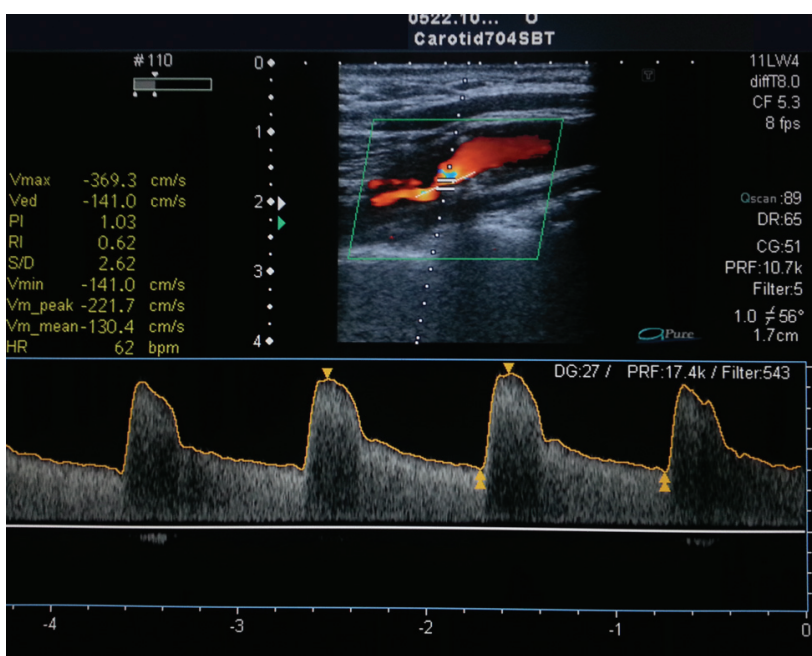

FIG 1. Patient 27 , with $\geq 70 \%$ stenosis of the left ICA. Duplex ultrasound image of the ICA shows a high PSV $(369 \mathrm{~cm} / \mathrm{s})$ and color flow turbulence immediately distal to the stenotic segment, broadening of the pulse wave Doppler spectrum, and a high end-diastolic velocity $(141 \mathrm{~cm} / \mathrm{s})$.

Patients with total or near-occlusion (defined as 0 PSV and no visible flow total or near-occlusion) were excluded.

Fifty-two patients with $50 \%-69 \%(n=20$, group 1$)$ and $70 \%-$ $99 \%(n=32$, group 2$)$ ICA stenosis were treated with protected CAS following a prospective protocol.

\section{Carotid Angiography and Carotid Artery Stent Placement Protocol}

All procedures were performed by the femoral approach by using the standard Judikins technique. At least 2 projections of the carotid artery stenosis were obtained for the calculations of vessel diameter and degree of stenosis.

CAS was performed by an experienced interventional cardiologist by using a 0.014 -inch platform with a distal embolus protection device (Emboshield NAV6; Abbott Laboratories, Abbott Park, Illinois). The appropriate length and diameter of self-expandable carotid stents (Xact; Abbott Laboratories) were used throughout the study. All stents were implanted from the ICA to the CCA. In case of technical difficulties with primary stent placement, the lesion was predilated before stent placement. After deployment, all stents were dilated with a 5.0- or $6.0-\mathrm{mm}$ balloon (Falcon Grande; Invatec, Roncadelle, Italy). The filter was retrieved through a $4.3 \mathrm{~F}$ dedicated catheter. A completion angiogram was obtained after removing the protection device. Patients were monitored in the recovery room and, barring any complication, were discharged in 3 days. The procedure was performed by the same experienced operator by using the same stent and the same filter (Fig 2), to keep any potential bias as low as possible.

\section{MR Imaging Protocol}

MR imaging was performed 48 hours before and after stent placement and at any time in case of neurologic deterioration (1.5T whole-body scanner, with a dedicated head coil; Signa Horizon; GE Healthcare, Milwaukee, Wisconsin).

DWI scans and the apparent diffusion coefficient maps were used to detect new acute ischemic lesions. A new lesion after CAS was defined as a focal hyperintense area detected by the fluid- 

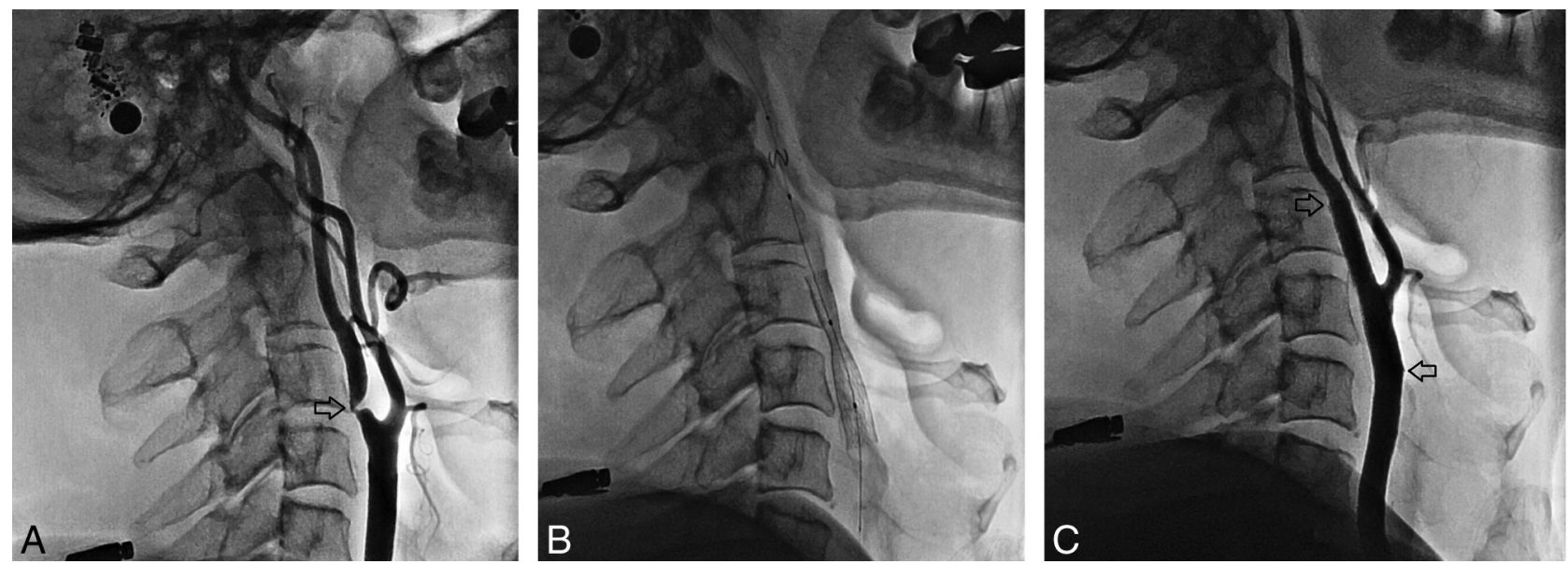

FIG 2. Patient 15. Angiographic sequence of carotid stent placement (a 65-year-old male patient who presented with right hemiplegia and hemiparesis). A, Baseline angiography (arrow indicates the ICA and CCA) shows $80 \%$ ICA stenosis. B, A filter was placed. The stent was then extended from the ICA into the CCA. C, Angiography after the procedure (arrows indicate ICA patency).
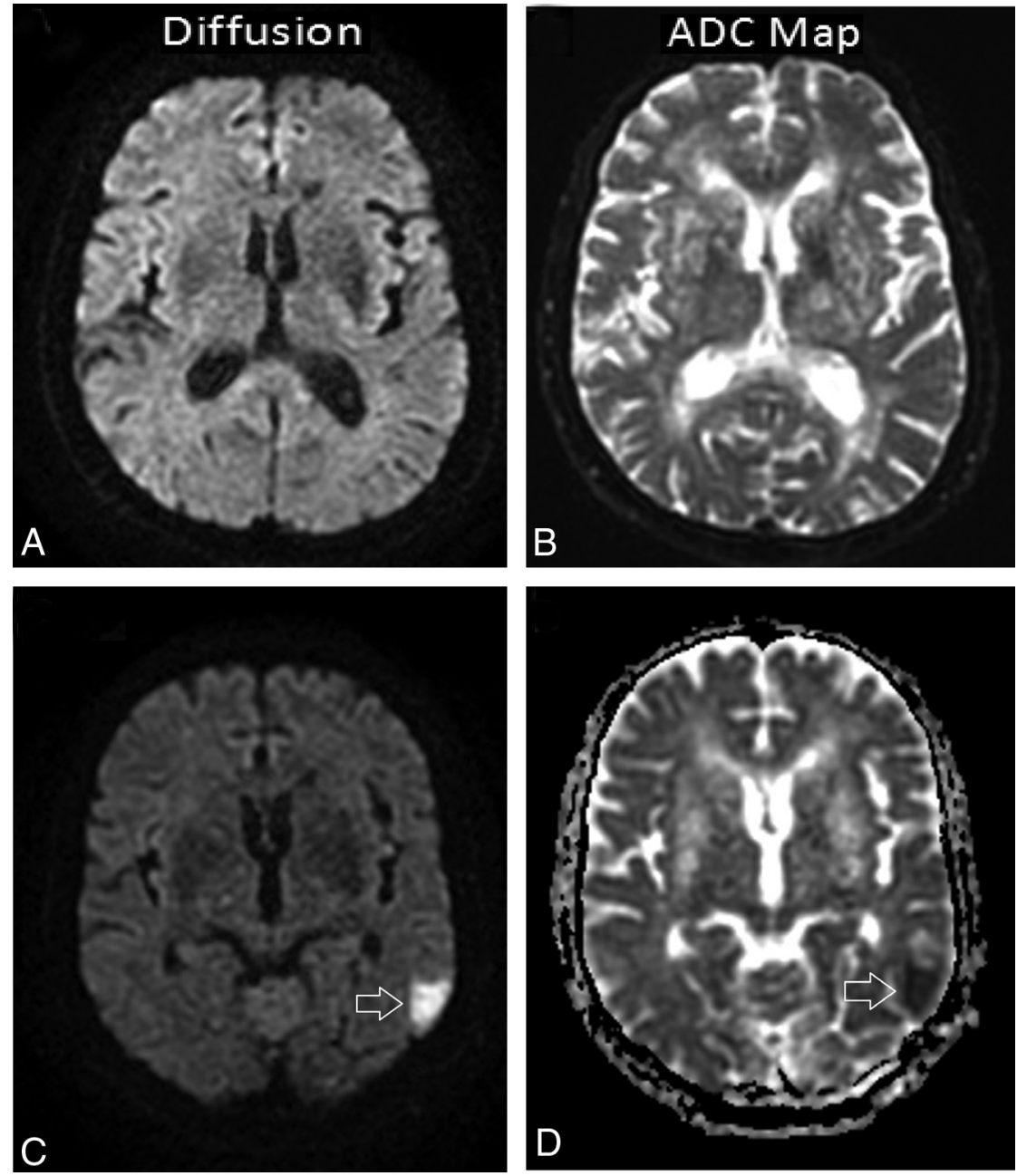

FIG 3. Patient 9 , a 58 -year-old woman with asymptomatic stenosis ( $\geq 80 \%$ ) of the right ICA. DWI and ADC map images in the 2 days before and the second day after filter-protected stent placement. $A$ and $B$, No visible lesion is seen on both images. $C$ and $D$, Poststent images demonstrate a single new ischemic lesion in the contralateral temporoparietal cortex (the lesion was hyperintense on the DWI and hypointense on the corresponding ADC map, arrowhead). attenuated inversion recovery sequence, corresponding to a restricted diffusion signal in the DWI sequence and confirmed by apparent diffusion coefficient mapping to rule out shinethrough artifacts. The diffusion-weighted sequence was performed with 2 levels of diffusion sensitization: $b=0$ and $b=1000 \mathrm{~s} / \mathrm{mm}^{2}$. The higher level of diffusion sensitization was replicated in each of the 3 principal gradient directions ( $x, y$, and $z$ planes). The diffusion-weighted images from each of the 3 diffusion-sensitized acquisitions were separately displayed for analysis. Besides DWI, other sequences, which comprised the axial spin-echo T1-weighted image, the fast spin-echo T1-weighted image, the fast spin-echo T2-weighted image, the FLAIR image, the perfusion image, and the contrast-enhanced spinecho T1-weighted image, were included (Fig 3). On each scan, the number and distribution (cortical, subcortical, or deep white matter) of lesions were recorded. Lesions were classified into 3 groups according to size ( $<5 \mathrm{~mm}, 5-10 \mathrm{~mm},>10$ $\mathrm{mm})$.

Two radiologists counted and recorded the number of new high-signal intensities in the cerebral hemispheres by comparing the pre- and postprocedural DWIs for each CAS procedure without any knowledge of the clinical status.

\section{Concomitant Medical Therapy}

All patients were treated with aspirin, 100 $\mathrm{mg} /$ day, plus clopidogrel, $75 \mathrm{mg} / \mathrm{day}$, for at least 7 days before CAS and 1 month 
Table 1: Baseline characteristics of study patients

\begin{tabular}{lc}
\hline \multicolumn{1}{c}{ Baseline Characteristics } & ( $\boldsymbol{n}=\mathbf{5 2}$ ) \\
\hline Mean age (yr) & $68 \pm 11(53-81)$ \\
Male/Female (No.) & $37: 15$ \\
Diabetes mellitus (\%) & 32 \\
Smoking (\%) & 42 \\
Hypertension (\%) & 66 \\
Dyslipidemia (\%) & 77 \\
Symptomatic (\%-No.) & $75-39$ \\
Coronary artery disease (\%) & 48 \\
Internal carotid artery, right ( $n$ ) & 24 \\
50-69\% Stenosis (treated vessel) & 20 \\
$\geq 70 \%$ Stenosis & 32 \\
Mean stenosis, preprocedure (\%) & $81 \pm 21$ \\
Mean stenosis, postprocedure (\%) & $12 \pm 7$ \\
DWI lesion count per patient & $2.8 \pm 1.2$ \\
(preprocedural) & \\
DWI lesion count (new) per patient & $3.4 \pm 0.7$ \\
(postprocedural) & \\
Mean lesion length (mm) & $16.4 \pm 5.1$ \\
Mean duration of procedure (min) & $29 \pm 12$ \\
\hline
\end{tabular}

afterward. During CAS, patients received intravenous heparin (5000-10,000 U) to maintain activated clotting time between 250 and 300 seconds. Just before the poststenting dilation phase, atropine (0.5-1 mg IV) was given to most patients to reduce bradycardia and hypotension potentially associated with carotid dilation.

\section{Statistical Analysis}

Statistical evaluation was performed by using the Statistical Package for the Social Sciences, Version 17.0, software package for Windows (IBM, Armonk, New York). Quantitative variables are given as mean $\pm \mathrm{SD}$, and qualitative variables are expressed as frequency and percentage. Groups were compared by using the Student $t$ test for continuous variables and the $\chi^{2}$ test for categoric variables. When $>2$ groups were compared for parameters, analysis of variance was used; post hoc analysis was performed by using the Tukey honest significant difference test. Logistic regression models were fitted for new brain lesions detected by DWI-MR frequency and extension as the dependent variable, with adjustment for Doppler parameters of ICA, age, sex, smoking status, hypertension, diabetes mellitus, and hyperlipidemia. For all analyses, a 2-tailed $P \leq .05$ was considered statistically significant.

\section{RESULTS}

In this prospective study, we also analyzed angiographic carotid stenosis on the basis of the North American Symptomatic Carotid Endarterectomy Trial criteria. ${ }^{17}$ Seventy-two patients underwent elective stent placement in the ICA, of which 52 consecutive patients were enrolled and 20 patients were excluded from the study.

The mean age of the patients was $68 \pm 11$ years (range, 53-83 years). Coronary artery disease was present in $48 \%$ of the patients $(n=25), 66 \%(n=29)$ had a history of hypertension, $32 \%(n=$ $18)$ had diabetes mellitus, $77 \%(n=40)$ had a history of dyslipidemia, $42 \%(n=22)$ were smokers, and 75\% $(n=39)$ were symptomatic. The demographic and clinical characteristics of the patients are presented in Table 1.

Among the patients who underwent carotid stent placement,
Table 2: Comparison of pre- and postprocedural Doppler flow parameters of ICA and CCA

\begin{tabular}{lccc}
\hline & $\begin{array}{c}\text { Preprocedural } \\
\text { Doppler Flow }\end{array}$ & $\begin{array}{c}\text { Postprocedural } \\
\text { Doppler Flow }\end{array}$ & $\boldsymbol{P}$ Value \\
\hline ICA PSV $(\mathrm{cm} / \mathrm{s})$ & $290 \pm 57$ & $77 \pm 24$ & $<.001$ \\
ICA EDV $(\mathrm{cm} / \mathrm{s})$ & $109 \pm 31$ & $34 \pm 10$ & $<.001$ \\
ICA-CCA ratio & $4.0 \pm 2.1$ & $1.4 \pm 0.9$ & $<.001$ \\
CCA PSV $(\mathrm{cm} / \mathrm{s})$ & $121 \pm 41$ & $107 \pm 15$ & .098 \\
CCA EDV (cm/s) & $31 \pm 11$ & $28 \pm 7$ & .441 \\
CCA RI & $0.75 \pm 0.38$ & $0.77 \pm 0.38$ & .631 \\
\hline
\end{tabular}

Note:- Rl indicates resistive index.

the preprocedural PSV, EDV, and ICA/CCA ratio in the treated internal carotid artery were $290 \pm 57 \mathrm{~cm} / \mathrm{s}, 109 \pm 31 \mathrm{~cm} / \mathrm{s}$, and $4.0 \pm 2.1$; and after stent placement, they were $77 \pm 24 \mathrm{~cm} / \mathrm{s}, 34 \pm$ $10 \mathrm{~cm} / \mathrm{s}$, and $1.4 \pm 0.9$, respectively. In addition, the preprocedural PSV, EDV, and resistive index in the ipsilateral CCA were $121 \pm 41 \mathrm{~cm} / \mathrm{s}, 31 \pm 11 \mathrm{~cm} / \mathrm{s}$, and $0.75 \pm 0.38$, whereas after stent placement, they were $107 \pm 15 \mathrm{~cm} / \mathrm{s}, 28 \pm 7 \mathrm{~cm} / \mathrm{s}$, and $0.77 \pm$ 0.38 , respectively. A marked decrease in ICA flow parameters was determined, and there was a statistically significant change from before to after ipsilateral stent placement; however, CCA flow parameters (PSV, EDV, and resistive index) did not change after ipsilateral ICA stent placement (Table 2).

When groups 1 and 2 were compared, the treated internal carotid artery PSV, EDV, and ICA/CCA ratio $(212 \pm 25 \mathrm{~cm} / \mathrm{s}$, $77 \pm 19 \mathrm{~cm} / \mathrm{s}, 3.3 \pm 0.9$, respectively) were significantly lower in group 1 than in group $2(351 \pm 61 \mathrm{~cm} / \mathrm{s}, 133 \pm 38 \mathrm{~cm} / \mathrm{s}, 4.8 \pm 1.9$, respectively). In contrast, the symptomatic patients' number, side of intervention, and carotid plaque length were not significantly different in group 1 versus group 2 patients (Table 3 ). In addition, sex, hypertension, diabetes, dyslipidemia, and smoking were not significantly different between the 2 groups.

Before the procedure, diffusion-weighted MR images revealed cerebral lesions in 39 (75\%) of 52 cases. The average number of preprocedural lesions was 2.8 per patient. After the procedure, new ipsilateral lesions were seen in $29(55.7 \%)$ of 52 cases, and new contralateral lesions were seen in 4 (7.6\%). The average number of new postprocedural lesions was 3.4 per patient. Eighteen (54.5\%) postprocedural lesions had a diameter of $<5 \mathrm{~mm}, 8$ $(24.2 \%)$ had a diameter of 5-10 mm, and $7(21.2 \%)$ had a diameter of $>10 \mathrm{~mm}$ on diffusion-weighted images. The size of the lesions in these patients varied from 3 to $23 \mathrm{~mm}$. Twenty-one $(63.6 \%)$ postprocedural lesions occurred in the cortical and subcortical areas of the brain, and $12(36.4 \%)$ postprocedural lesions were in the deep brain areas. In 29 patients, these new lesions were clinically silent. Four patients, who had new lesions on postprocedural MR images, experienced adverse neurologic events during or immediately after the intervention: 2 patients had a minor stroke, 1 patient had a transient ischemic attack, and 1 patient had transient monocular blindness. There were no deaths or major strokes during the procedure.

When groups 1 and 2 were compared, pre- and postprocedural DWI lesion count per patient and number of patients with new lesions $(1.4 \pm 0.7,2.2 \pm 19 \mathrm{~cm} / \mathrm{s}, 7$, respectively) were significantly lower in group 1 than in group $2(5.1 \pm 0.9,4.7 \pm 2.3 \mathrm{~cm} / \mathrm{s}$, 26 , respectively). In contrast, symptomatic patients' number, side of intervention, and carotid plaque length did not differ significantly between group 1 and group 2 patients (Table 3 ). Univariate 
Table 3: Comparison of patients with ICA stenosis $50 \%-69 \%$ and $>70 \%$; correlation of preprocedural Doppler flow parameters of carotid stenosis and new ischemic brain lesions on DWI after internal carotid artery stenting

\begin{tabular}{|c|c|c|c|c|c|}
\hline \multirow[b]{2}{*}{ Characteristics } & \multirow{2}{*}{$\begin{array}{c}50 \%-69 \% \text { Stenosis } \\
(n=20)\end{array}$} & \multirow{2}{*}{$\begin{array}{l}\geq 70 \% \text { Stenosis } \\
\quad(n=32)\end{array}$} & \multirow{2}{*}{$\begin{array}{c}\text { Univariate Analysis, } \\
\qquad \text { Value }\end{array}$} & \multicolumn{2}{|c|}{ Multivariate Analysis } \\
\hline & & & & OR & $P$ Value \\
\hline ICA PSV (cm/s) & $212 \pm 25$ & $351 \pm 61$ & $<.001$ & 1.421 & $<.001$ \\
\hline ICA EDV (cm/s) & $77 \pm 19$ & $133 \pm 38$ & .003 & 1.024 & .021 \\
\hline ICA-CCA ratio & $3.3 \pm 0.9$ & $4.8 \pm 1.9$ & .007 & 1.009 & .038 \\
\hline Symptomatic (\%, No.) & $55 \%-11$ & $87.5 \%-28$ & .169 & - & - \\
\hline Patients with new lesions (No.) & 7 & 26 & .013 & - & - \\
\hline DWI lesion count per patient (preprocedural) & $1.4 \pm 0.7$ & $5.1 \pm 1.9$ & $<.001$ & - & - \\
\hline DWI lesion count (new) per patient (postprocedural) & $2.1 \pm 0.9$ & $4.7 \pm 2.3$ & $<.001$ & - & - \\
\hline Side of intervention, right (No.) & 10 & 14 & - & - & - \\
\hline Mean lesion length (mm) & $14.8 \pm 3.8$ & $17.6 \pm 6.1$ & .451 & - & - \\
\hline
\end{tabular}

analysis revealed that there was no significant correlation between the occurrence of a new DWI lesion and either age, presence of $\mathrm{CAD}$, hypertension, diabetes mellitus, smoking, or hyperlipidemia $(P>.05)$. Multivariate analysis revealed a significant correlation between ICA flow parameters, especially PSV, and each of the DWI lesion counts per patient (pre- and postprocedural, Table 3 ).

Furthermore, technical success $(<30 \%$ post-CAS diameter stenosis) was achieved in all patients, and postprocedural angiography demonstrated a patent ICA in all patients (Fig 1). The percentage diameter stenosis of the ICA decreased from $81 \pm 21 \%$ preprocedure to $12 \pm 7 \%$ postprocedure. Neither dissection nor spasm severe enough to produce ICA flow impairment was observed.

\section{DISCUSSION}

We found that Doppler flow parameters (especially PSV) of internal carotid artery stenosis were significantly correlated with periprocedural ischemic events associated with carotid stent placement. In contrast, known atherosclerotic risk factors (age, presence of CAD, hypertension, diabetes mellitus, and hyperlipidemia) were not significantly associated with new DWI lesions. This finding suggests that PSV is an important flow parameter, more specifically applying to stenosis, and a predictive marker of ischemic cerebral lesions.

Several investigators, by using DWI to detect clinically silent embolic lesions after protected CAS, have reported new DWI lesions over a wide range, from $17.3 \%$ to $73 \%{ }^{20}$ Furthermore, the results from transcranial Doppler monitoring and experimental studies show that embolization may occur in nearly all stent-implantation procedures in the carotid artery. ${ }^{21}$ The rate of new DWI lesions in our study was similar compared with these other studies. Of 52 patients, $63 \%$ had new lesions after CAS with distal filter protection, and new ischemic lesions were detected in the treated vascular territory in $55 \%$ of the patients. Because the protective function of filters is limited to treated vessels, and a certain number of embolic events ipsi- and contralateral to the treated vessels are probably sourced from endovascular procedures, ${ }^{13}$ these findings cast doubt on the efficacy of the routine use of filter-type cerebral protection devices.

Clearly, the more complete evaluation of brain injury performed at least 48 hours postprocedure is to be preferred if one is examining the incidence of new lesions on DWI. ${ }^{22,23}$ We also used diffusion-weighted MR imaging within 48 hours before and after the procedure. The mean number of new lesions inside the treated vascular territory in our study (3.4 per patient) was higher compared with the reported incidence of between 1.42 and $2.8 .^{13,20} \mathrm{In}$ agreement with these results, most were at the cortical and corticosubcortical area and $<5 \mathrm{~mm}$. The clinical importance of asymptomatic cerebral microemboli is still unclear. ${ }^{16,21}$ In our series, most of (29 of 33) the patients with new postprocedural DWI lesions were neurologically asymptomatic, and only the neurologic status of 4 patients changed within 48 hours after CAS.

In patients with carotid stenosis, microembolism has been related to the degree of carotid narrowing and to the ulcerated appearance of the plaque. ${ }^{6,24,25}$ Sterpetti et $\mathrm{al}^{26}$ examined prospectively 214 consecutive patients referred to a vascular laboratory and found that a degree of stenosis of $>50 \%$ was an independent predictor of new cerebrovascular events. Until now, to our knowledge, no study has been published that specifically evaluated the relationship between DWI findings after protected CAS and Doppler flow parameters of ICA. We found a significant relationship between ICA Doppler flow parameters, especially PSV, and the appearance of new lesions. Moreover, patients with $\geq 70 \%$ stenosis had a significantly higher number of new lesions compared with patients with $50 \%-69 \%$ stenosis. The most plausible reason for the relation between the frequency of ischemic lesions and Doppler flows of the stenotic ICA segment is that the higher the flow of stenotic ICA, the more plaque formation and solid emboli source.

The present study shows that known cardiovascular risk factors such as age, blood pressure, and cholesterol levels were not significantly related to ischemic cerebrovascular events detected by DWI. However, PSV, EDV, and the ICA/CCA ratio and preand postprocedural DWI lesions per patient were significantly different in univariate analysis. Most interesting, in multivariate analysis, these Doppler parameters were significant predictors of cerebrovascular events. Thus, in our opinion, an accurate determination of Doppler flow data of carotid artery stenosis may be useful to plan medical or/and interventional therapy for the primary as well as for secondary prevention of cerebrovascular events.

\section{Limitations}

This is a single-center study. In line with our finding, the use of cerebral protection devices does not appear to significantly reduce the number of new ipsilateral DWI lesions after CAS. The number of patients, however, was too small to allow investigation by using 
a full regression model, though association between the occurrence of new DWI lesions and Doppler flow characteristics and cross-validation may be needed.

\section{CONCLUSIONS}

Our data showed that CAS is associated with a high number of new ischemic lesions at cerebral MR imaging, of which most are clinically silent. We believe that the use of Doppler parameters plays a crucial role in the accurate assessment of the degree of ICA stenosis and in the prediction of new ischemic DWI lesions. The examination of a larger patient population and the analysis of Doppler flow parameters showing varying degrees of stenosis may allow identification of patients who have a high rate of positive findings on postprocedural diffusion-weighted images.

\section{REFERENCES}

1. Poungvarin N. Stroke in the developing world. Lancet 1998; 352(suppl 3):SIII19-22

2. Alam I, Haider I, Wahab F, et al. Risk factors stratification in $\mathbf{1 0 0}$ patients of acute stroke. J Postgrad Med Inst 2004;18:583-91

3. Strickman NE, Loyalka P. Carotid artery stenosis: an endovascular specialist's perspective. Tex Heart Inst J 2005;32:318-22

4. Siebler M, Sitzer M, Rose G, et al. Silent cerebral embolism caused by neurologically symptomatic high-grade carotid stenosis. Brain 1993;116:1005-15

5. Siebler M, Kleinschmidt A, Sitzer M, et al. Cerebral microembolism in symptomatic and asymptomatic high-grade internal carotid artery stenosis. Neurology 1994;44:615-18

6. Sitzer M, Muller W, Siebler M, et al. Plaque ulceration and lumen thrombus are the main sources of cerebral microemboli in highgrade internal carotid artery stenosis. Stroke 1995;26:1231-33

7. Heijenbrok-Kal MH, Nederkoorn PJ, Buskens E, et al. Diagnostic performance of duplex ultrasound in patients suspected of carotid artery disease: the ipsilateral versus contralateral artery. Stroke 2005;36:2105-09

8. Ota H, Takase K, Rikimaru H, et al. Quantitative vascular measurements in arterial occlusive disease. Radiographics 2005;25:1141-58

9. Shaikh NA, Bhatty S, Irfan M, et al. Frequency, characteristics and risk factors of carotid artery stenosis in ischaemic stroke patients at Civil Hospital Karachi. J Pak Med Assoc 2010;60:8-12

10. Tahmasebpour HR, Buckley AR, Cooperberg PL, et al. Sonographic examination of the carotid arteries. Radiographics 2005;25:1561-75

11. Demarin V, Lovrencić-Huzjan A, Basić S, et al. Croatian Society for Neurovascular Disorders; Croatian Society of Neurology; Croatian Society of Ultrasound in Medicine and Biology; Croatian Society of Radiology; Croatian Society of Vascular Surgery; Croatian Society of Neurosurgery: recommendations for the management of patients with carotid stenosis. Acta Clin Croat 2010;49:101-18

12. Liapis CD, Bell PR, Mikhailidis D, et al, for the ESVS Guidelines Collaborators. ESVS guidelines: invasive treatment for carotid stenosis: indications, techniques. Eur J Vasc Endovasc Surg 2009; 37(4 Suppl):1-19

13. Piñero P, González A, Mayol A, et al. Silent ischemia after neuroprotected percutaneous carotid stenting: a diffusion-weighted MRI study. AJNR Am J Neuroradiol 2006;27:1338-45

14. Marks MP, De Crespigny A, Lentz D, et al. Acute and chronic stroke: navigated spin-echo diffusion-weighted MR imaging. Radiology 1996;199:403-08

15. González RG, Schaefer PW, Buonanno FS, et al. Diffusion-weighted MR imaging: diagnostic accuracy in patients imaged within 6 hours of stroke symptom onset. Radiology 1999; 210:155-62

16. Bendszus M, Koltzenburg M, Burger R, et al. Silent embolism in diagnostic cerebral angiography and neurointerventional procedures: a prospective study. Lancet 1999;354:1594-97

17. Britt PM, Heiserman JE, Snider RM, et al. Incidence of postangiographic abnormalities revealed by diffusion-weighted MR imaging. AJNR Am J Neuroradiol 2000;21:55-59

18. North American Symptomatic Carotid Endarterectomy Trial Collaborators. Beneficial effect of carotid endarterectomy in symptomatic patients with high-grade carotid stenosis. $N$ Engl J Med 1991;325:445-53

19. Grant EG, Benson CB, Moneta GL, et al. Carotid artery stenosis: gray-scale and Doppler US diagnosis-Society of Radiologists in Ultrasound Consensus Conference. Radiology 2003;229:340-46

20. Stojanov D, Ilic M, Bosnjakovic P, et al. New ischemic brain lesions on diffusion-weighted MRI after carotid artery stenting with filter protection: frequency and relationship with plaque morphology. AJNR Am J Neuroradiol 2012;33:708-14

21. Jaeger HJ, Mathias KD, Hauth E, et al. Cerebral ischemia detected with diffusion-weighted MR imaging after stent implantation in the carotid artery. AJNR Am J Neuroradiol 2002;23:200-07

22. Kastrup A, Nagele T, Groschel K, et al. Incidence of new brain lesions after carotid stenting with and without cerebral protection. Stroke 2006;37:2312-16

23. du Mesnil de Rochemont R, Schneider S, Yan B, et al. Diffusionweighted MR imaging lesions after filter-protected stenting of high-grade symptomatic carotid artery stenoses. AJNR Am J Neuroradiol 2006;27:1321-25

24. Eicke B, von Lorentz J, Paulus W. Embolus detection in different degrees of carotid disease. Neurol Res 1995;17:181-84

25. Valton L, Larrue V, Arrué P, et al. Asymptomatic cerebral embolic signals in patients with carotid stenosis: correlation with appearance of plaque ulceration on angiography. Stroke 1995;26:813-15

26. Sterpetti AV, Schultz RD, Feldhaus RJ, et al. Ultrasonographic features of carotid plaque and the risk of subsequent neurologic deficits. Surgery 1988;104:652-60 